\title{
Tapentadol: what every doctor needs to know about this new drug
}

\author{
Tapentadol: o que todo médico precisa saber sobre esse novo fármaco
}

Bernardo De Marchi Mosele, Daniel Benzecry de Almeida², Válery Baggio Hess ${ }^{3}$

DOI 10.5935/2595-0118.20180015

\section{ABSTRACT}

BACKGROUND AND OBJECTIVES: Acute and chronic pain is a major problem with repercussion in our society, causing impairment in the quality of life of patients as well as socioeconomic losses, due to work absenteeism. This review aims to analyze the use of tapentadol, an analgesic not yet available in Brazil, with a dual mechanism of action, in the treatment of acute and chronic pain syndromes.

CONTENTS: A review about this new drug was made on the Pubmed database using the keywords "tapentadol" and "opioids," evaluating its pharmacological and clinical aspects comparing with other current drugs in pain treatment, as well as its indications and contraindications in the management of patients with pain disorders.

CONCLUSION: Tapentadol has been shown to be effective in the treatment of acute and chronic pain, with potency equivalent to the opioids currently used. In addition, it developed less tolerance, less adverse effects and better therapeutic response in chronic neuropathic pain when compared to pure $\mu$-opioid receptors agonists.

Keywords: Dual mechanism of action, Opioids, Tapentadol.

\section{RESUMO}

JUSTIFICATIVA E OBJETIVOS: A dor aguda e crônica consiste em um problema de grande repercussão em qualquer sociedade atual, causando degradação na qualidade de vida dos próprios pacientes e comprometimento socioeconômico pelo absenteísmo laboral. O objetivo deste estudo foi analisar o uso do tapentadol, um analgésico ainda náo disponível no Brasil e com duplo mecanismo de ação, no tratamento das síndromes dolorosas agudas e crônicas.

\footnotetext{
1. Santa Casa de Santos, Centro de Ensino e Treinamento em Anestesiologia, Santos, SP, Brasil. 2. Instituto de Neurologia de Curitiba, Grupo de Tratamento de Dor, Curitiba, PR, Brasil. 3. Hospital Estadual Guilherme Álvaro, Departamento de Residência Médica em Anestesiologia, Santos, SP, Brasil.

Submitted in August 15, 2017

Accepted for publication in January 05, 2018.

Conflict of interests: none - Sponsoring sources: none

Correspondence to:

Instituto de Neurologia de Curitiba, Grupo de Tratamento de Dor

Rua Jeremias Maciel Perretto, 300 - Campo Comprido - Ecoville

81210-310 Curitiba, PR, Brasil.

E-mail: bmosele@gmail.com

(C) Sociedade Brasileira para o Estudo da Dor
}

CONTEÚDO: Foi realizada uma pesquisa na base de dados Pubmed utilizando os descritores "tapentadol" e "opioides" para revisão da literatura mundial sobre esse novo fármaco, avaliando as características farmacológicas e aspectos clínicos do seu uso na realidade atual, em comparação com os fármacos já existentes no mercado, assim como suas indicaçóes e contraindicações no manuseio do paciente com dor.

CONCLUSÁO: O tapentadol se mostrou eficaz no tratamento de dores agudas e crônicas, com potência equiparável aos opioides já comercializados. Além disso, desenvolveu menos tolerância, menos efeitos adversos e melhor resposta terapêutica na dor crônica neuropática quando comparado com agonistas receptores opioides tipo $\mu$ puros.

Descritores: Duplo mecanismo de ação, Opioides, Tapentadol.

\section{INTRODUCTION}

Pain is a problem of great proportion, involving 20 to $25 \%$ of the population with an increasing incidence. Despite the extraordinary advances in anatomy, physiology, diagnosis and pain management, more than $50 \%$ of patients report improper relief. This can be related to the neuroplasticity process of the pain pathways ${ }^{1}$, creating a persistent "memory" of the aggressive event and neuronal hiperexcitability ${ }^{2,3}$. Understanding chronic pain neurobiology is fundamental to target the treatment. Thus, the use of drugs that act on more than one pathophysiologic mechanism, as tapentadol, can be a new weapon in the therapeutic armamentarium against acute pain and especially chronic ones. The objective of this study was to analyze the use of tapentadol, an analgesic not yet available in Brazil, with a dual mechanism of action, in the treatment of acute and chronic pain syndromes.

\section{CONTENTS}

Tapentadol is a single non-racemic molecule (Figure 1) with a dual central mode of action, available in the United States of America (USA) and in Europe for the treatment of acute and chronic pain $^{1.4}$. It is in available in the U.S. since 2008 for the treatment of moderate acute to severe pain, in its immediate release (IR) formulation, and since 2011 for the treatment of moderate chronic to severe pain in its extended release (ER) formulation, after approval by the Food and Drug Administration (FDA) ${ }^{5,6}$. In the European market, the IR and ER formulations of the drug were approved by the European Medicines Agency (EMA) in 2010

Its central analgesic action occurs by means of two mechanisms: 1 ) as an agonist of the $\mu$-opioid receptors (MOR), with the same affinity or 10 times higher on KOR receptors (k-opioid receptor) 


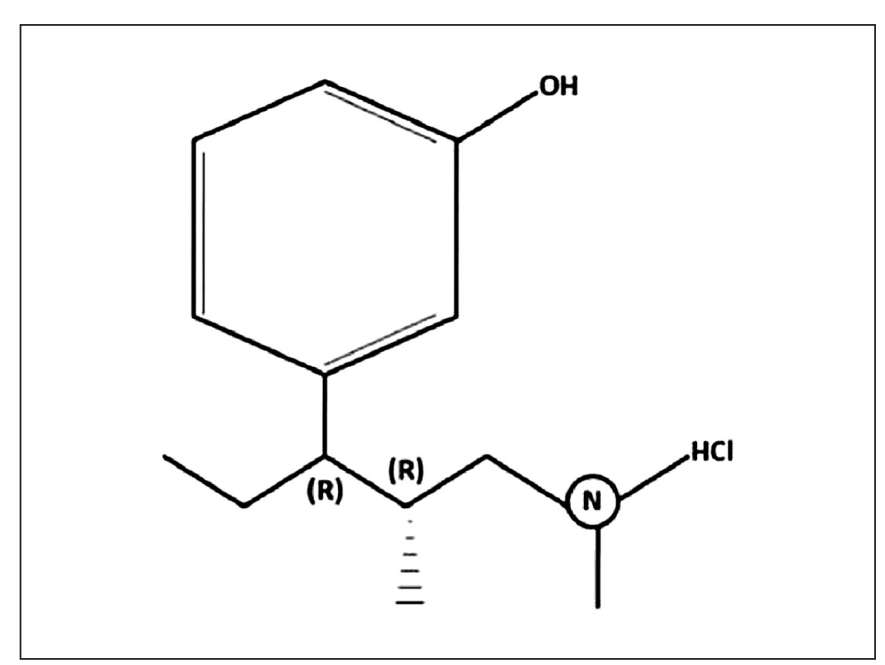

Figure 1. Molecular formula $\mathrm{C}_{14} \mathrm{H}_{23} \mathrm{NO} \cdot \mathrm{HCl}^{4}$

and DOR (delta opioid receptor) ${ }^{4} ; 2$ ) as a norepinephrine reuptake inhibitor (NRI). The MOR affinity is considered modest when compared to pure agonists, such as morphine ${ }^{9,10}$, whose affinity is 44 to 50 times higher ${ }^{2,4}$.

Its NRI action increases the norepinephrine release by the pain descending inhibitory pathways in the dorsal horn of the spinal cord, allowing the substance to accumulate in the synapses. Norepinephrine binds to the alpha ${ }_{2}$-adrenergic inhibitors of the pre- and post-synaptic neurons, inhibiting the action potential by MOR identical mechanisms (Figure 2), blocking the presynaptic calcium channels and activating the post-synaptic potassium channels, hyperpolarizing the membrane ${ }^{1}$. In models of visceral, neuropathic and inflammatory pain, all of acute and chronic nature, the NRI dose-dependent action was reported ${ }^{11}$. It was observed, in vitro, a weak action on the serotonin reuptake with no apparent analgesic effect ${ }^{4,9}$.

It has fast oral absorption, reaching its maximum serum concentration in less than 2 hours, and can be taken disregarding meals ${ }^{12,13}$. The bioavailability is of $32 \%$ due to first pass effect. Plasma concentration in stationary state occurs in approximately $25-30$ with the administration at every 6 hours ${ }^{4,11,14}$. After in-

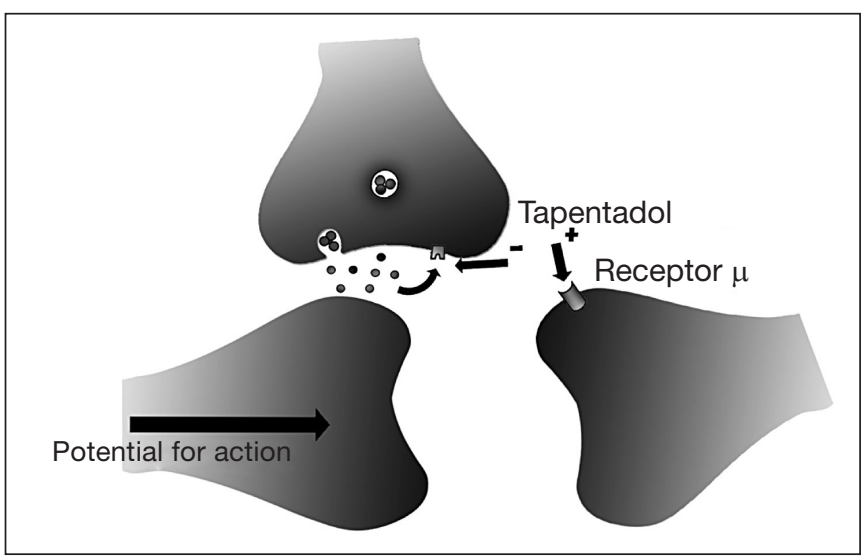

Figure 2. Agonist mechanism of action on $\mu$-opioid receptors and inhibitory action on norepinephrine reuptake (MOR-NRI) travenous administration, it is widely distributed, and only $20 \%$ bind to plasma proteins $s^{4}$.

The main metabolic pathway occurs through glucuronic acid binding, $97 \%$ due to phase 2 reactions. The main metabolite is tapentadol-O-glucuronide ${ }^{4,8}$, which does not exercise any activity on opioid receptors or reuptake systems of synapses or other junction'. Ninety seven percent of the drug delivered is transformed into inactive metabolites ${ }^{4}$. No significant induction or inhibition of the $\mathrm{P} 450$ cytochrome (CYP) was reported ${ }^{11}$. There was no relevant clinical change in the pharmacokinetic properties when delivered concomitantly with non-opioids, as well as with omeprazole and metoclopramide ${ }^{10,11}$. The elimination halflife is of approximately 4 hours ${ }^{9}$, and the metabolites are excreted in 24 hours and completely eliminated in 5 days, almost apart through the kidneys. The fecal excretion is negligible, approximately $1 \%$, $42,14,15$.

Tapentadol IR is available in 50,75 and $100 \mathrm{mg}$ tablets. The recommended dose is at every 4-6h, depending on criteria such as the intensity of pain, tolerability, and response to treatment. Total doses higher than $700 \mathrm{mg}$ in the first day or $600 \mathrm{mg}$ in the subsequent days were not studied ${ }^{6}$.

Tapentadol ER is available in 50, 100, 150, 200 and 250mg tablets. In Spain, there is the $25 \mathrm{mg}$ presentation for more precise dose adjustments in senior and liver disease patients. The ER formulation is indicated for the treatment of moderate to severe chronic pain and cannot be used without a ceiling dose as the other opioids. It is recommended 100 to $250 \mathrm{mg} \mathrm{BD}$ (at every 12 hours $)^{16}$. In patients who had never used opioids, the initial dose is $50 \mathrm{mg}$. For patients taking another opioid, the initial dose will depend on the type and daily dose of the drug in use. In these cases, titration to increase the $50 \mathrm{mg}$ BD should occur every 3 days, until the adequate pain control. Due to the tolerability profile, especially the gastrointestinal, tapentadol ER allows for relatively fast titration with effective pain control. Daily doses higher than $500 \mathrm{mg}$ are not recommended. Like other formulations of extended release opioids, tablets shall not be broken or crushed $^{6,10}$.

Tapentadol IR presented great efficacy in acute pain after a bunionectomy and dental surgery, in back pain, and knee and hip joint pain. Tapentadol ER was also effective in chronic back pain, fibromyalgia, neuropathic and osteoarthritis-related pain $^{12,17,18}$. Also, it had benefits in injury and nerve inflammation models, with predictability in the management of bone cancer pain, a state of mixed, nociceptive and neuropathic pain ${ }^{19}$. Its broad therapeutic spectrum makes it effective in nociceptive and neuropathic acute and chronic pain'. Moreover, the synergic dual mechanism reduces the drug-drug interaction risk associated with the polypharmacy approach ${ }^{6}$.

Tapentadol was also effective in patients with hyperalgesia due to central sensitization who have already tried multiple subtypes of opioids ${ }^{5}$. It is suggested that the combination of tapentadol ER with pregabalin is well tolerated and effective, being this association more favorable than the pure agonist opioids ${ }^{10}$.

The relevance of the dual mechanism of action was investigated by combining tapentadol with naloxone or alpha ${ }_{2}$ yohimbine in models of acute and chronic pain. It was noted that the opi- 
oid agonist contributes to analgesia in acute pain, whereas the NRI action played a fundamental role in chronic pain ${ }^{2}$. Another important factor related to the NRI action is its usefulness in chronic neuropathic pain ${ }^{20}$. Additional evidence of the efficacy in states of neuropathic pain comes from a study in diabetic neuropathy models, in which tapentadol inhibited thermal hyperalgesia and morphine did not. Pure opioids lose power and efficacy in neuropathic pain due to an unsatisfactory therapeutic window, while the noradrenergic drugs become more efficient. Even being 2 to 3 times less potent than morphine in a series of acute models, tapentadol became at least equipotent in neuropathic models, ratifying the reason for opioids not be considered the first line of treatment for this type of pain and its use be preferred for refractory neuropathic pain $2,20,21$. The FDA approved the use of tapentadol for the treatment of diabetic neuropathic pain in 2012 due to its proved efficacy when compared to placebo. However, we still lack evidence of clinical relevance comparing tapentadol with first-line drugs, such as pregabalin, duloxetine, and tricyclic antidepressants ${ }^{22,23}$.

Another use of tapentadol would be in acute pain after an outpatient surgical procedure. Despite the improvements in protocols structure, pain - in the outpatient surgery context - remains a challenge due to the limitation of the analgesic techniques when compared with hospitalized patients. Pain killers with multimodal actions can improve the postoperative analgesia and the functionality of outpatient surgeries ${ }^{24}$.

The most frequent adverse effects reported associated with the continuous opioid therapy are a problem for patients with chronic pain, such as nausea in 6 to $42 \%$, vomit in 8 to $33 \%$ and constipation in 3,1 to $95 \%$ of patients ${ }^{18}$. The main adverse effects of tapentadol are gastrointestinal and central, the most common being nausea $(30 \%)$, followed by vomiting $(18 \%)$, drowsiness $(15 \%)$ and dizziness $(24 \%)$. Of these, the ones that have really led to treatment discontinuation were dizziness $(2.6 \%)$, nausea $(2.3 \%)$, vomiting $(1.4 \%)$, drowsiness $(1.3 \%)$ and headache $(0.9 \%)^{14,17}$. Tapentadol was associated with the reduction of $50 \%$ in treatment discontinuation when compared with similar drugs $^{7}$. On the other hand, there were more treatment discontinuations due to lack of effect than morphine, oxycodone, and fentanyl patch, but also fewer discontinuations due to the same reasons for placebo ${ }^{25}$. Another recognized adverse effect due to the continuous use of MOR agonists in men is the opioid-induced androgen deficiency, leading to erectile dysfunction, reduction in sperm counts, testicular atrophy, and hair rarefaction. Studies have shown that tapentadol presented a relatively lesser magnitude of these effects. Reports of reduction of some typical opioid-induced secondary adverse effects in comparison with doses of the same analgesic potency of classic opioids corroborate the hypothesis that this higher analgesic efficacy of tapentadol is only partially mediated by opioid agonist mechanisms, which are spared by the drug NRI action ${ }^{19}$.

Studies that evaluated the long-term tolerability, corroborated by meta-analysis results, indicated that tapentadol is not associated with laboratory alterations for liver or kidney function, and did not affect the ECG, blood pressure or heart rate parameters since the NRI action increases the chances of cardiovascular effects ${ }^{14,17,26}$.
As for the development of withdrawal symptoms, one must follow the same protocol as any other opioid, with gradual and regressive discontinuation. The symptoms after abrupt discontinuation of the drug are minimal ${ }^{10,11}$.

Tapentadol ER is well tolerated and effective up to 2 years of treatment in patients with chronic pain, with no development of acquired tolerance to the analgesic effects in this period ${ }^{10}$.

Concerning drug abuse rate, evidence from monitoring systems suggests low rate in the clinical practice ${ }^{10}$. During the first 24 months after the initial release and commercialization of tapentadol IR in the U.S., the abuse rates found were much lower than those for oxycodone or hydrocodone, through a system called RADARS $^{12}$. A cohort study showed that the risk of abuse with tapentadol was lower when compared to oxycodone $e^{8}$.

A study with rats and mice studied the acute toxicity by oral and venous administration. The average values of lethality were above $300 \mathrm{mg} / \mathrm{kg}$ for oral administration and $40 \mathrm{mg} / \mathrm{kg}$ for venous administration. The most common effects after the administration of high doses were central, such as sedation, excited behavior, and respiratory depression ${ }^{12}$. On the abuse by humans, a retrospective study compared the tapentadol toxicity with tramadol using the information of the U.S. National Poison Data System. The reasons for exposure include the non-intentional (iatrogenic and improper use) and intentional ones (suspicion of suicide and abuse). The exposure to tapentadol was associated with the significantly higher risk of a serious clinical outcome, like respiratory depression, coma, lethargy, slurred speech, hallucination, and confusion. Tramadol was associated with higher convulsion and vomiting rates. Although these drugs have similar mechanisms of action, tapentadol was associated with clinical effects significantly more toxic ${ }^{27}$. A case report was published about the death of a 34-year male after the use of endovenous tapentadol. The patient was found with gasping-like respiratory pattern ${ }^{28}$. In a cohort study carried out with children with an average age of 4.17 years, drowsiness and lethargy were the most observed adverse effects (28.8\%). More important adverse effects such as respiratory depression, coma and dyspnea only happened in $7.7 \%$ of the sample ${ }^{29}$.

To compare drugs with a dual mechanism of action, as tapentadol, it is essential to consider the analgesic equivalence and not the opioid equivalence ${ }^{19}$. Considered tapentadol closest competitor, since they share certain chemical superficial structural similarities ${ }^{30}$, tramadol also has more than one mechanism of action, with agonist action on opioid receptors, minimum NRI effect and significant effect in inhibiting serotonin reuptake (SSRI) ${ }^{6}$, binding to MOR, KOR and DOR with low affinity, being, therefore, less effective than other opioids to treat intense pain ${ }^{6,9}$. However, tapentadol is pharmacologically very different from tramadol. Many pharmacological interactions can be triggered by tramadol since some antiarrhythmic, antidepressants, antipsychotics, antiparasitic drugs and tamoxifen are a substrate for $\mathrm{CYP}^{9,24}$. In animal models, tapentadol showed to be 2 to 5 times more potent than tramadol. Even assuming a conservative conversion rate of $1: 2$ between tapentadol and tramadol based on the maximum daily recommended dose of $400 \mathrm{mg}$ for tramadol, this dose would reach only $40 \%$ of the analgesic effect of the maximum daily dose of tapentadol. Consequently, an intermediate daily dose of $250 \mathrm{mg}$ of tapentadol would 
extend over the effect of the maximum dose of tramadol. A factor that could explain the lower potency and clinical efficacy of tramadol, in spite of its affinity with MOR be more intense, would be the fact that tramadol does not cross the blood-brain barrier in the same ratio that its dose is increased, while tapentadol crosses it, following its concentration gradient ${ }^{2,30}$. Tapentadol showed a greater benefit in several pain scenarios, mainly due to the reduction of the pro-nociceptive effect of the neuropathies, the increase of norepinephrine in the dorsal horn of the spinal cord, and the reduction of the inconvenient adverse effects in similar analgesic doses, like nausea and vomiting, caused by the 5-HT serotonin receptor antagonist ${ }^{9}$.

Thus, we can conclude that the comparison between these drugs is inappropriate since their potency and analgesic efficacy are disproportional. This higher tapentadol efficacy led the majority of the studies to consider oxycodone as the benchmark ${ }^{30}$. Studies have shown that in the acute postoperative pain, the analgesic efficacy of the $50-75 \mathrm{mg}$ tapentadol IR is not inferior to oxycodone IR $10 \mathrm{mg}$ and that $100 \mathrm{mg}$ of tapentadol IR is equivalent to $15 \mathrm{mg}$ of oxycodone $\mathrm{IR}^{19,31}$. Another comparative study of 10 days on the management of acute back pain showed that tapentadol IR has analgesic efficacy and global security comparable to oxycodone IR for the relief of moderate to severe acute back pain, as well as more flexibility in the dose regimen to improve gastrointestinal tolerability, especially related to vomiting and constipation ${ }^{11,31}$. A systematic review used 7 clinical assays with a direct comparison between tapentadol ER 100-250mg BD and oxycodone ER 10$20 \mathrm{mg} \mathrm{BD}$, as well as the immediate release formulations, tapentadol IR, and oxycodone IR in the doses of 50-100mg versus 10$15 \mathrm{mg}$ every 4-6 hours, respectively, to manage severe chronic pain. At the end of the treatment, significant differences were found in pain relief, between 30 and 50\% favoring tapentadol, as well as a significant advantage of tapentadol over oxycodone in terms of patient's general impression and improvement of the quality of life. The grouped analysis on the number of serious adverse events showed boundary significance for tapentadol over oxycodone. With regard to specific adverse events, there were significant differences favoring tapentadol with respect to the incidence of constipation, nausea, and vomiting. Oxycodone groups showed a lower incidence of discontinuation to due lack of efficacy, while treatment discontinuation for any reason and adverse events were significantly more frequent $t^{25}$. The superiority of tapentadol over oxycodone can, in part, to be related to the higher tolerability, reducing the number of patients who discontinue the treatment, mainly during dose titration. It is possible to establish a total therapeutic effect more frequently with tapentadol.

Tapentadol ER showed higher tolerability compared to morphine, specifically in the reduction of the incidence of nausea, vomiting, dizziness, and constipation ${ }^{10}$. Its analgesic potency in nociceptive pain models would be around 2,5 times lower than morphine ${ }^{2}$. In animal models with equianalgesic doses of tapentadol and morphine, the complete tolerance was significantly decreased in the tapentadol group ( 23 days) in comparison to the morphine group (10 days), what seems to be due to its dual mode of action. The first group also showed a lower physical dependence $^{2,15}$. Unlike morphine, tapentadol keeps its efficacy after a neuronal injury. Neuropathy powers its actions in low doses, although its affinity with MOR is smaller?

Tapentadol is prohibited in patients with paralytic ileum, impaired pulmonary function or in places with no monitoring or resuscitation equipment ${ }^{6}$. In obese patients with obstructive sleep apnea syndrome, caution should be even higher. The concomitant use of central nervous system depressants can foster the effects of sedation, respiratory depression and decreased vigilance ${ }^{10}$. Due to the NRI action, with the NRI coadministration, norepinephrine reuptake inhibitors, tricyclic antidepressants and primarily with monoamine oxidase inhibitors (MAOI) may trigger cardiovascular effects such as hypertensive crises and even serotonin syndrome, being contraindicated the use of MAOI in the last 14 days $^{6,11}$.

The incidence of chronic pain proportionally increases with the age $^{23}$. Dose adjustment is not required with tapentadol $\mathrm{ER}^{10}$. However, it is advisable to start with low doses in patients older than 65 years $^{4}$.

It is not recommended in patients with less than 18 years of age ${ }^{10,11}$, pregnant and breastfeeding women (category C) ${ }^{11}$, with severe liver or kidney failure ${ }^{6}$. Dose adjustment in liver and kidney failure is not required ${ }^{4,6,10,17}$.

Tapentadol has unique pharmacological characteristics. Some authors suggest that it should be considered the first of a new pharmacologic class, the MOR-NRI ${ }^{20,30}$. It was the first analgesic with MOR and NRI agonist action released in the global market. It showed to be effective in the treatment of innumerable situations of acute and chronic pain, with good tolerability and low potential of pharmacological interactions, becoming the preferred choice in the pain control in the outpatient setting ${ }^{11}$.

It is worth mentioning the major limitations as to the quantity and quality of clinical trials. In addition, the manufacturer has been responsible for, or the sponsor of many of studies and review articles published ${ }^{23}$.

The main advantages are the analgesic potency comparable to pure agonist opioids in the treatment of nociceptive, neuropathic and mixed types of chronic pain, as well as the tolerability profile due to the low incidence of gastrointestinal and central adverse effects and the prolonged relief of the symptoms with a low addiction rate ${ }^{10,16}$.

\section{CONCLUSION}

Since tapentadol maximizes the advantages of the multimodal therapy, mitigates the adverse effects and drug interactions, its entry in the Brazilian market should be evaluated by the competent agencies.

\section{ACKNOWLEDGMENTS}

To Mrs. Marli Uchida for the revision and Dr. Erasmo Barros da Silva Júnior for providing figure 2 .

\section{REFERENCES}

1. Fornasari D. Pain pharmacology: focus on opioids. Clin Cases Miner Bone Metab. 2014;11(3):165-8.

2. Pergolizzi J, Alegre C, Blake D, Alén JC, Caporali R, Casser HR, et al. Current con- 
siderations for the treatment of severe chronic pain: the potential for tapentadol. Pain Pract. 2012;12(4):290-6.

3. Hartrick CT, Rozek RJ. Tapentadol in pain management a $\mu$-opioid receptor agonist and noradrenaline reuptake inhibitor. CNS Drugs. 2011;25(5):359-70.

4. Singh DR, Nag K, Shetti AN, Krishnaveni N. Tapentadol hydrochloride: a novel analgesic. Saudi J Anaesth. 2013;7(3):322-6.

5. Mercadante S, Porzio G, Gebbia V. New Opioids. J Clin Oncol. 2014;32(16):1671-6.

6. Hartrick CT, Rodríguez Hernandez JR. Tapentadol for pain: a treatment evaluation. Expert Opin Pharmacother. 2012;13(2):283-6.

7. Santos J, Alarcáo J, Fareleira F, Vaz-Carneiro A, Costa J. Tapentadol for chronic musculoskeletal pain in adults. Cochrane Database Syst Rev. 2015;(5):CD009923.

8. Vadivelu N, Kai A, Maslin B, Kodumudi G, Legler A, Berger JM. Tapentadol extended release in the management of peripheral diabetic neuropathic pain. Ther Clin Risk Manag. 2015;11:95-105.

9. Rodriguez MA. Tapentadol, una nueva opción terapêutica. Rev Col Anest. 2011;39(3):375-85.

10. Sánchez Del Águila MJ, Schenk M, Kern KU, Drost T, Steigerwald I. Practical considerations for the use of tapentadol prolonged release for the management of severe chronic pain. Clin Ther. 2015;37(1):94-113.

11. Wade WE, Spruill WJ. Tapentadol hydrochloride: a centrally acting oral analgesic. Clin Ther. 2009;31(12):2804-18.

12. Knezevic NN, Tverdohleb T, Knezevic I, Candido KD. Unique pharmacology of tapentadol for treating acute and chronic pain. Expert Opin Drug Metab Toxicol. 2015;11(9):1475-92.

13. Hartrick CT. Tapentadol immediate-release for acute pain. Expert Rev Neurother. 2010;10(6):861-9.

14. Frampton JE. Tapentadol immediate release: a review of its use in the treatment of moderate to severe acute pain. Drugs. 2010;70(13):1719-43.

15. Pierce DM, Shipstone E. Pharmacology update: tapentadol for neuropathic pain. Am J Hosp Palliat Care. 2012;29(8):663-6.

16. Taylor R, Pergolizzi JV, Raffa RB. Tapentadol extended release for chronic pain patients. Adv Ther. 2013;30(1):14-27.

17. Hoy SM. Tapentadol extended release: in adults with chronic pain. Drugs. 2012;72(3):375-93
18. Afilalo M, Morlion B. Efficacy of tapentadol ER for managing moderate to severe chronic pain. Pain Physician. 2013;16(1):27-40.

19. Langford RM, Knaggs R, Farquhar-Smith P, Dickenson AH. Is tapentadol different from classical opioids? A review of the evidence. Br J Pain. 2016;10(4):217-21.

20. Tzschentke TM, Cristoph T, Kögel BY. The mu-opioid receptor agonist/noradrenaline reuptake inhibition (MOR-NRI) concept in analgesia: the case of tapentadol. CNS Drugs. 2014;28(4):319-29.

21. Hartrick CT. Tapentadol immediate release for the relief of moderate-to-severe acute pain. Expert Opin Pharmacother. 2009;10(16):2687-96.

22. Desai B, Freeman E, Huang E, Hung A, Knapp E, Breunig IM, et al. Clinical value of tapentadol extended-release in painful diabetic peripheral neuropathy. Expert Rey Clin Pharmacol. 2014;7(2):203-9.

23. Veal FC, Peterson GM. Pain in the frail or elderly patient: does tapentadol have a role? Drugs Aging. 2015;32(6):419-26.

24. Nossaman VE, Ramadhyani U, Kadowitz PJ, Nossaman BD. Advances in perioperative pain management: use of medications with dual analgesic mechanisms, tramadol \& tapentadol. Anesthesiol Clin. 2010;28(4):647-66.

25. Riemsma R, Forbes C, Harker J, Worthy G, Misso K, Schafer M, et al. Systematic review of tapentadol in chronic severe pain. Curr Med Res Opin. 2011;27(10):1907-30

26. Vadivelu N, Timchenko A, Huang Y, Sinatra R. Tapentadol extended-release for treatment of chronic pain: a review. J Pain Res. 2011;4:211-8.

27. Tsutaoka BT, Ho RY, Fung SM, Kearney TE. Comparative toxicity of tapentadol and tramadol utilizing data reported to the national poison data system. Ann Pharmacother. 2015;49(12):1311-6.

28. Kemp W, Schlueter S, Smalley E. Death due to apparent intravenous injection of tapentadol. J Forensic Sci. 2013;58(1):288-91.

29. Borys D, Stanton M, Gummin D, Drott T. Tapentadol toxicity in children. Pediatrics. 2015;135(2):392-6

30. Raffa RB, Buschmann H, Cristoph T, Eichenbaum G, Englberger W, Flores CM, et al. Mechanistic and functional differentiation of tapentadol and tramadol. Expert Opin Pharmacother. 2012;13(10):1437-49.

31. Biondi D, Xiang J, Benson C, Etropolski M, Moskovitz B, Rauschkolb C. Tapentadol immediate release versus oxycodone immediate release for treatment of acute low back pain. Pain Physician. 2013;16(3):E237-46. 